NASZA DERMATOLOGIA Onlin OUR DERMATOLOGY Online

Source of Support: Nil

Competing Interests: None

\section{TUBERCULAR ABSCESS OF THE LOWER LIP: A RARE CASE OF MISTAKEN IDENTITY}

\author{
Safia Rana ${ }^{1}$, Seema Monga ${ }^{2}$, Sabina Khan ${ }^{1}$, Shaan Khetrapal ${ }^{1}$, \\ Sujata Jetley ${ }^{1}$
}

${ }^{I}$ Department of Pathology, Hamdard Institute of Medical Sciences and Research, Jamia Hamdard, Hamdard Nagar, New Delhi 110062, India

${ }^{2}$ Deparment of ENT, Hamdard Institute of Medical Sciences and Research, Jamia

Hamdard, Hamdard Nagar, New Delhi 110062, India

Corresponding author: Prof. Sujata Jetley

sujatajetley@gmail.com

\begin{abstract}
Tuberculosis of the oral cavity accounts for less than one percent of all cases of tuberculosis, seen in both the primary and secondary stages of the disease. It presents usually as a single, painful, ulcer however, multiple painless ulcers may also be seen. The most common location in the oral cavity is tongue. The palate, buccal mucosa, floor of the mouth, gingiva, and lip are other possible sites of involvement. In secondary tuberculosis, oral lesions are accompanied by lesions in the lungs, lymph nodes, or in any other part of the body. We hereby report a case of tuberculosis of lip in a 24 year old female, secondary to pulmonary tuberculosis.
\end{abstract}

Key words: Tuberculosis; lip; secondary

\section{Introduction}

Oral lesions of tuberculosis can be seen either as primary lesion or may manifest as secondary stage of the disease. The commonest site of tubercular involvement in the oral cavity is the tongue and involvement of lip, as was seen in this case is exceptionally rare. Primary oral tuberculosis may present as a diagnostic challenge for the clinician especially when it is the the sole manifestation of the disease [1]. Secondary oral tuberculosis usually results from inoculation by the infected sputum or hematogenous spread by Mycobacteria [2].

\section{Case Report}

A 24 year old female patient presented in the ENT Outpatient Department with lower lip swelling for past 25 days. There was no history of fever, cough or hemoptysis. The patient complained of loss of appetite and weight since the last few months. On enquiring, a history of contact was elicited, as her husband was a known case of tuberculosis who was diagnosed 3 years back. A history of irregular intake of anti-tubercular drugs was also obtained from him. The patient was a nonsmoker and there was no history of alcohol intake or tobacco chewing. Her general condition was stable, she was afebrile and no pallor, icterus or cervical lymphadenopathy was noted. Local examination showed a swelling on the mucosal aspect of the lower lip which had a soft cystic consistency, measuring $1 \mathrm{x} 1 \mathrm{~cm}$ in size and reddish blue in colour (Fig. 1). A provisional diagnosis of retention cyst was made in the ENT OPD and patient was referred for fine needle aspiration cytology of the lip swelling. Aspiration yielded a blood mixed fluid aspirate which was stained with Giemsa stain and examined microscopically. The FNAC smears showed moderate cellularity comprising of sheets of histiocytes along with neutrophils in a highly necrotic background. Many epithelioid cell granulomas and multinucleated giant cells were also evident (Fig. 2). Ziel Neilson stain for acid fast bacilli was found to be positive (Fig. 3 ). A cytological impression of tubercular abscess, lower lip was made.

Routine haematological and biochemical investigations were all within normal limits however Erythrocyte Sedimentation Rate was raised with a $40 \mathrm{~mm}$ fall in 1 st hour. Routine urine and microscopy was within normal limits. Serological tests for Hepatitis B Surface Antigen, HIV-1 and HIV-2 were nonreactive. 
Chest X ray PA view showed a lobulated soft tissue opacity in the right hilum suggestive of lymphadenopathy and also small opacities in right upper lobe. CECT chest was advised for further evaluation. CECT thorax showed multiple enlarged, conglomerate, necrotic peripherally enhancing lymph nodes in right paratracheal, pretracheal, subcarinal and prevascular, right hilar locations. Few small discrete nodular opacities with surrounding ground glass haze were seen in the posterior

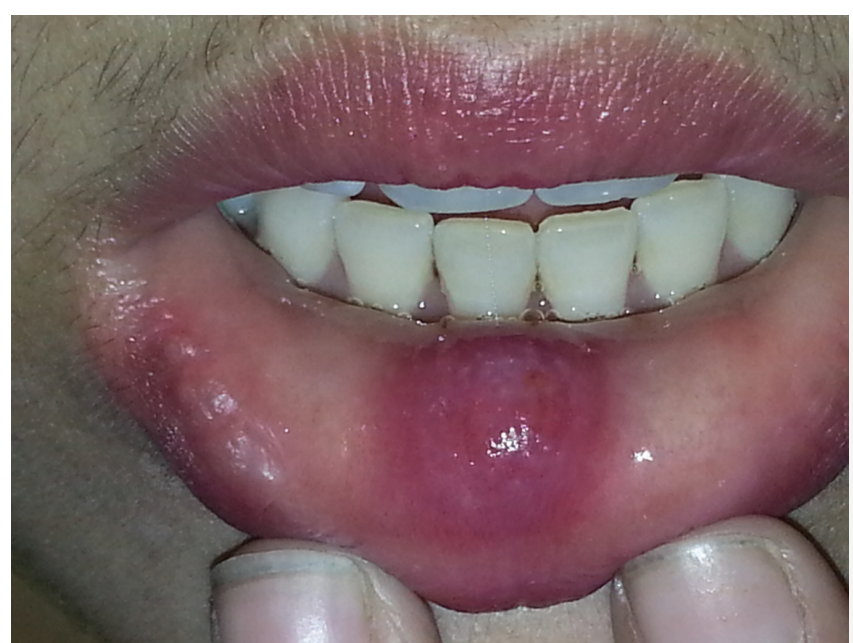

Figure 1. A swelling on the mucosal aspect of lower lip.

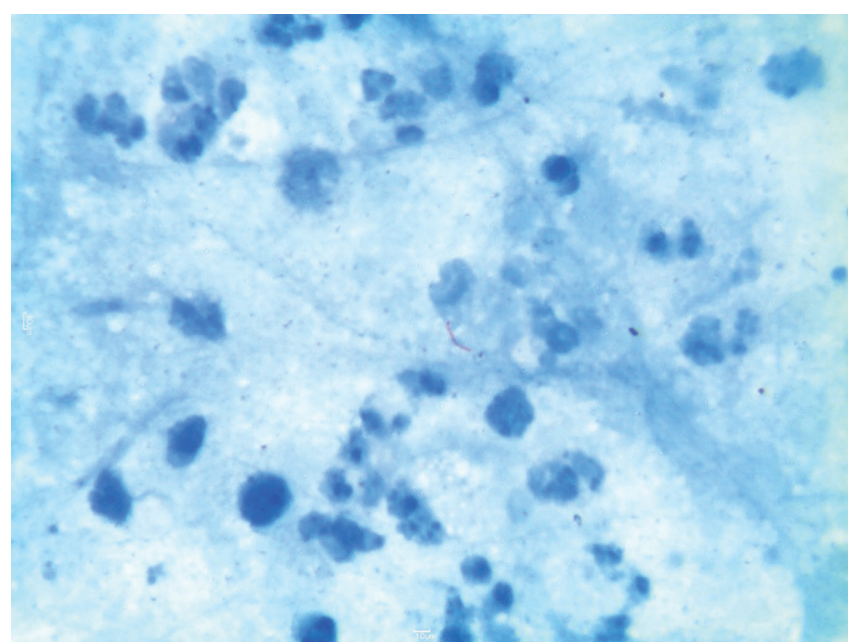

Figure 3. Presence of acid fast bacilli. (Z.N stain, 100X)

\section{Discussion}

The oral cavity is an uncommon site of involvement by tuberculosis. Oral TB is rare and accounts for less than $1 \%$ of all cases of TB. However with increase in number of TB cases, these unusual forms of oral cavity are more likely to occur and may be misdiagnosed [3]. Oral tuberculosis is seldom primary, but more commonly secondary to pulmonary disease. A study has reported four cases of tuberculosis of oral cavity one of which was primary and three were secondary. [4] It was segment of right upper lobe, subpleural in right base, apical segment of left lower lobe and in left basal region. The radiological impression of an infective pathology, likely Koch's etiology was made A. standard combination treatment of four drug regimen (anti-tuberculous treatment) was started, which resulted in symptomatic improvement in the patient and healing of the lesion.

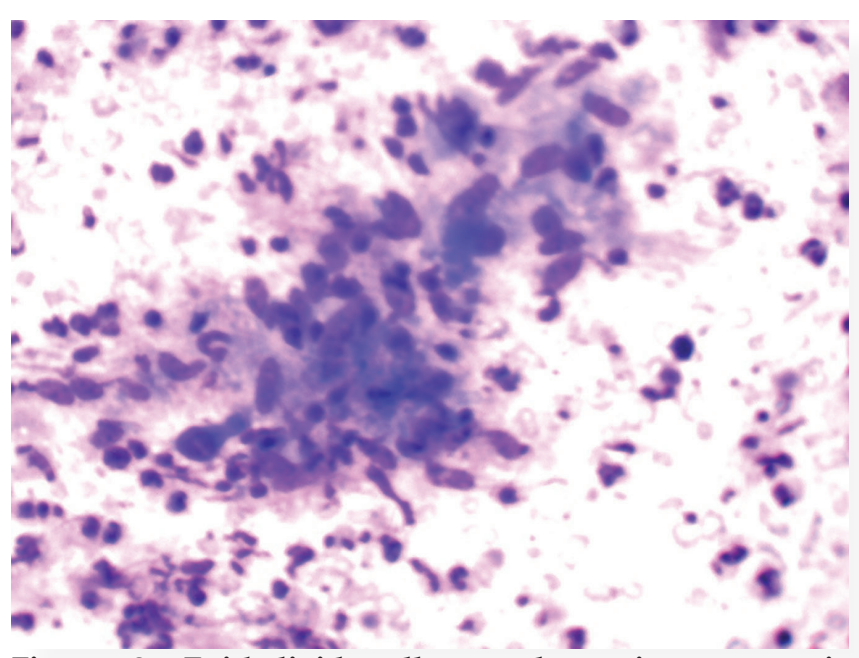

Figure 2. Epithelioid cell granuloma in a necrotic background. (Giemsa, 40X)

found as a secondary infection in $58 \%$ and as primary infection in $42 \%$ patients [5]. Oral lesions of tuberculosis are nonspecific in their clinical presentation and are often overlooked by the clinician. Tuberculous lesion occasionally precedes the detection of pulmonary tuberculosis as seen in the present case. Mostly lesion presents as a non healing painful ulcer [5] in which a differential diagnosis of simple traumatic ulcer and carcinoma can also be considered. In the present case the clinical presentation was unusual, a cystic lesion was seen in the lower lip and the first impression was a retention cyst. There were no enlarged cervical lymphnodes which also was an unusual feature.

M. tuberculosis infects all parts of the oral cavity, more often in males [5]. The tongue, gingiva and palate are the most frequent sites of involvement by oral tuberculosis. In the tongue the common sites of ulcer formation are the lateral border and the tip of the tongue, whereas the hard palate is more frequently involved than the soft palate [1]. Tubercular involvement of the lip, as seen in our case is even rarer.

Although the exact pathogenesis, is still unknown, the organism enters through breach in the mucosal surface. Abbot et al were able to isolate the tubercle bacilli from mouth washings of $44.9 \%$ of the patients with active pulmonary tuberculosis [6]. It is usually acquired through infected sputum coughed out by a patient with open pulmonary tuberculosis or by hematogenous spread [7]. The tubercle bacilli are transferred from a primary focus in some part of the body and localized in oral cavity, after trauma. The systemic factors include impaired host resistance and increased virulence of the organisms [8]. 
The involvement of maxilla and the mandible may occur secondary to the extension of gingival lesion or by hematogenous route leading to tuberculous osteomyelitis. FNAC suggested the diagnosis of a granulomatous lesion and the diagnosis of tuberculosis was considered. It was further confirmed by AFB staining. FNAC may show similar findings in atypical mycobacterial infections, sarcoidosis and lymphomas [9].

In oral cavity $\mathrm{TB}$, standard treatment regimen is successful, so a planned standard combination treatment of four drug regimen was started, resulting in gradual relief of symptoms and healing of the lesion $[10,11]$.

\section{Conclusion}

Tubercular involvement of the lip is a rare presentation of a common disease. This case highlights the importance of a high index of suspicion among clinicians for this manifestation of tuberculosis which will lead to timely diagnosis and institution of specific anti-tubercular treatment. Evidence of systemic or lung involvement may not be present in all cases and laboratory and radiological findings serve as a good marker, especially when there is high index of suspicion. Definitive tissue diagnosis along with demonstration of $\mathrm{AFB}$, as in the present case remains the gold standard.

\section{REFERENCES}

1. Ebenezer J, Samuel R, Mathew GC, Koshy S, Chacko RK, Jesudason MV. Primary oral tuberculosis: Report of two cases. Ind J Dent Res. 2006;17:41-4.

2. Smith WHR, Davis D, Mason KD, Onions JP. Intraoral and pulmonary tuberculosis following dental manipulation. Lancet. 1982;1:842-3.

3. Nanda KD, Mehta A, Marwaha M, Kalra M, Nanda JA. Disguised tuberculosis in oral buccal mucosa. Dent Res J. 2011;8:154-9.

4. Dogra SS, Chander B, Krishna M. Tuberculosis of oral cavity: a series of one primary and three secondary cases. Indian J Otolaryngol Head Neck Surg. 2013;65:275-9.

5. Kakisi OK, Kechagia AS, Kakisis IK, Rafailidis PI, Falagas ME. Tuberculosis of the oral cavity: a systematic review. Eur J Oral Sc. 2010;118:103-9.

6. Abbot JN, Briney AT, Denaro SA. Recovery of Tubercle bacilli from mouth washings of tuberculous dental patients. J Am Dent Assoc. 1955;50:49-52.

7. Tuberculosis cutis orificialis (TBCO): a rare manifestation of tuberculosis. Chauhan V, Mahesh DM, Panda P, Mahajan S, Thakur S. J Assoc Physicians India. 2012;60:126-7.

8. Sharma P, Saxena S, Aggarwal P, Reddy V. Tuberculosis of odontogenic cyst. Indian J Tuberc. 2013;60:50-4.

9. Sareen D, Sethi A, Agarwal AK. Primary tuberculosis of the tongue: a rare nodular presentation. Br Dent J. 2006;200:321-2.

10. Cawson RA, Binnie WH, Barrett AW, Wright JM. Mucocutaneous diseases and other forms of stomatitis. Oral Disease, 3rd edition. Mosby, London. 2001:13.7.

11. Çakan A, Mutlu Z, Ozsoz A, Erbaycu AE, Ünal T, Koyuncu BO. Tuberculosis of oral mucosa. Monaldi Arch Chest Dis. 2001;5:315-7.

Copyright by Safia Rana, et al. This is an open access article distributed under the terms of the Creative Commons Attribution License, which permits unrestricted use, distribution, and reproduction in any medium, provided the original author and source are credited. 